2017-11-15

Understanding the psychosocial needs of pituitary patients: A survey of Pituitary Foundation members.

\title{
Norman, Alyson
}

http://hdl.handle.net/10026.1/10256

All content in PEARL is protected by copyright law. Author manuscripts are made available in accordance with publisher policies. Please cite only the published version using the details provided on the item record or document. In the absence of an open licence (e.g. Creative Commons), permissions for further reuse of content should be sought from the publisher or author. 


\section{Abstract}

Background: Management of pituitary conditions is notoriously difficult and patients often experience high levels of distress which are often not addressed by health professionals. As a result, pituitary conditions have been found to have a large negative impact on quality of life. The aims of this study were 1) to identify the psychosocial symptoms associated with pituitary conditions, and 2) to identify any differences in symptomatology across different pituitary conditions, age ranges and gender.

Methods: A questionnaire was completed by 1062 members of the Pituitary Foundation (683 female, aged under 18 to over 65). With a format based on the Cancerbackup Survey (2006), the questionnaire was designed using material from the Pituitary Foundation Needs Analysis report (2006) and some relevant questions from other validated questionnaires. Respondents

completed either a pen and paper version or went online to survey monkey.

Results: Physical and psychosocial issues were identified, including mood swings, fatigue, anxiety and depression. The results identified significant variation in symptoms across gender, age range and condition type, particularly in relation to infertility and headaches difficulties with sex life, appearance and pain management. Headaches were associated with women, younger patients and those with prolactinoma, diabetes insipidus, acromegaly or hypogonadism. Difficulties with

sex life were associated with men, and those with prolactinoma.

Conclusions: Participants were found to be experiencing a range of debilitating psychosocial and physical symptoms that were impairing long term functioning. These symptoms need further support in terms of patient information, advice and condition management.

\section{Objectives}

Management of Pituitary conditions is notoriously difficult and patients often experience high levels of distress which are often not addressed by health professionals ${ }^{1}$. As a result, pituitary conditions have been found to have a large negative impact on quality of life ${ }^{1}$.

Part of the difficulty in understanding the effect pituitary conditions can have on patients is the lack of research looking at the needs of patients with pituitary conditions and how they manage their care ${ }^{2}$. Further research is needed to identify the extent of the psychosocial needs of patients by mapping symptoms associated with poor quality of life and identifying any differences in presentation across pituitary conditions.

\section{Aims:}

This study had the following aims: 1)identify the psychosocial symptoms associated with pituitary conditions, and

2)identify any differences in

symptomatology across different pituitary conditions, age ranges and gender.

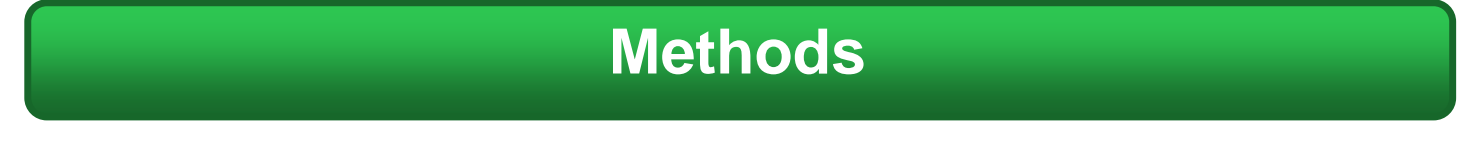

Results
Participants: A questionnaire was sent to 2,000 members of the Pituitary Foundation. Of the total sample approached, 1062

responded, a response rate of $53 \%$. 683 respondents were female and 366 were male (13 unknown), with an age range of under 18 years of age to over 65

Patients had a range of pituitary conditions including hypopituitarism (473), diabetes insipidus (183), nonfunctioning tumour (170), acromegaly (138), prolactinoma (123), Cushing's disease (98), craniopharyngioma (64), hypogonadism (367) and Sheehan's syndrome (14).

Design: The questionnaire was designed using material from a needs analysis of pituitary patients and relevant questions taken from other validated questionnaires. The format was based upon the Cancerbackup survey (2006) ${ }^{3}$

Patients were asked to list the types of psychosocial symptoms they had experienced as a result of their pituitary condition.

Procedure: Respondents were asked to complete either a pen and paper version of the questionnaire or to complete the questionnaire online using survey monkey.
The survey identified difficulties with physical pain, extreme fatigue and fluctuating mood. Half of participants identified that their condition had affected their ability to work and reduced their fertility. increased levels of anxiety and depression and changes in appearance. Other psychosocial issues included poor quality of sleep and poor sex life.

Regression analyses showed infertility was associated with younger patients $\left(r^{2}=-0.207\right.$, $\mathrm{p}<0.001)$, and those with hypopituitarism $\left(r^{2}=0.164, p<0.001\right)$ or prolactinoma $\left(r^{2}=0.117, p<0.001\right)$.

Headaches were associated with younger patients $\left(r^{2}=-0.165\right.$, $p<0.001)$, female patients $\left(r^{2}=0.128\right.$, $\mathrm{p}<0.001)$, and those with prolactinoma $\left(r^{2}=0.159, p<0.001\right)$, Diabetes Insipidus $\left(r^{2}=0.083\right.$, $p<0.01)$, acromegaly $\left(r^{2}=0.063\right.$, $\mathrm{p}<0.05)$ and hypogonadism $\left(\mathrm{r}^{2}=\right.$ $0.074, p<0.05)$.

Poor satisfaction with the quality of sex life was associated with male patients $\left(r^{2}=0.112, p<0.001\right)$, and those with prolactinoma $\left(r^{2}=0.068\right.$, $\mathrm{p}<0.05)$.
Participants also highlighted

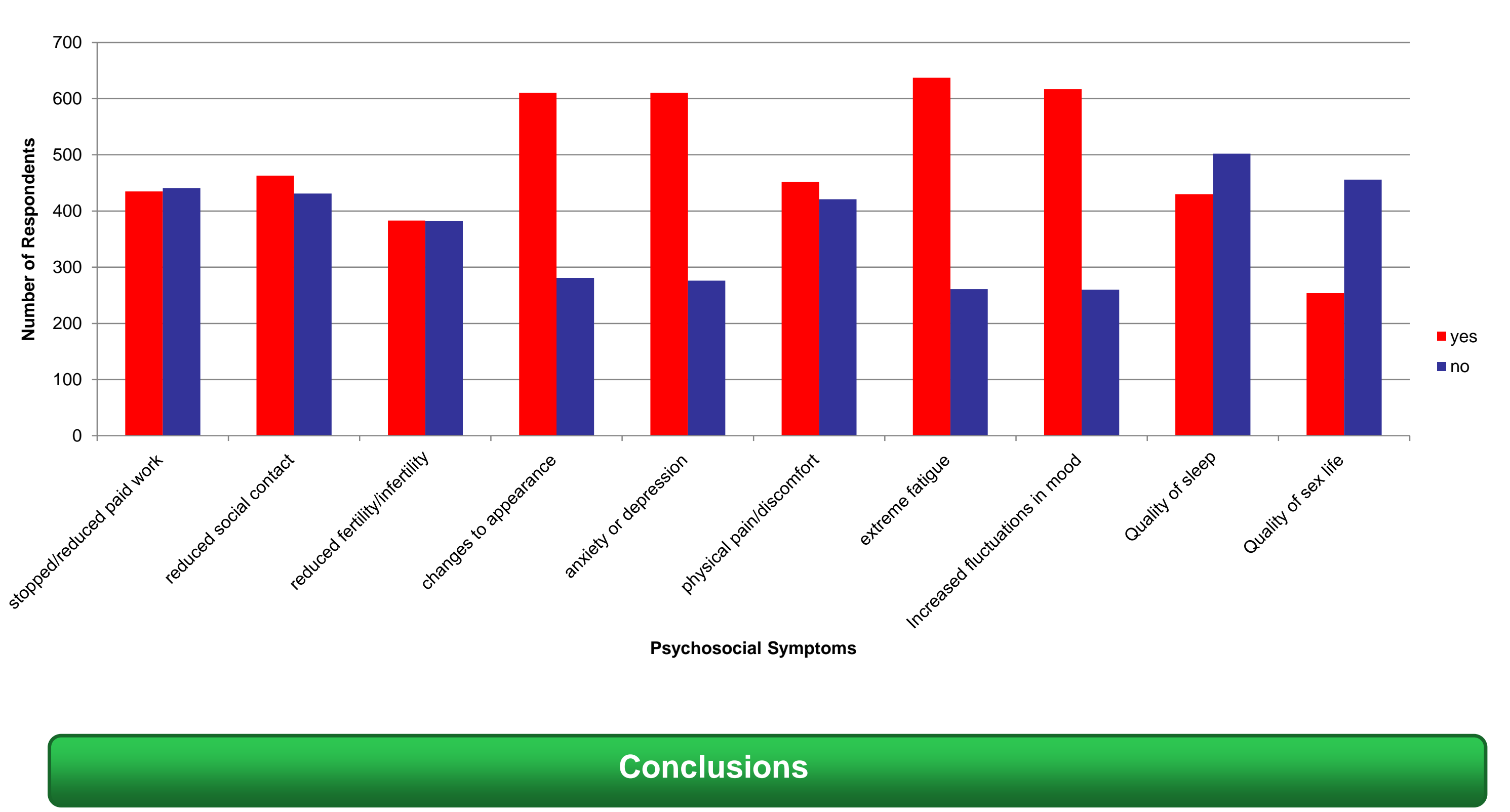

Pituitary conditions have a negative impact on quality of life. Participants were found to be experiencing a range of debilitating psychosocial and physical symptoms that were impairing long term functioning.

These symptoms need further support in terms of patient information, advice and condition management.

\section{References}

1. Osbourne, M., Jackson, S., Morris , M., \& Ashley, K. (2006). Cinderella's story: the psychosocial impact of pituitary conditions. Endocrine Abstracts, 12, 84.

2. Jackson, S., Murray, J., Morris, M., Woods, T. (2008). Patient Satisfaction Survey final report for Pituitary Foundation. Pituitary Foundation.

3. Cancerbackup (2006). Big cancer information survey. www.cancerbackup.org.uk. Accessed 03.09.2015. 